\title{
Energy Turnaround: Printing of Thermoelectric Generators
}

\author{
Ines Dani ${ }^{1}$, Aljoscha Roch ${ }^{1}$, Lukas Stepien ${ }^{1}$, Christoph Leyens ${ }^{1,2}$, \\ Moritz Greifzu², Marian v. Lukowicz ${ }^{2}$ \\ ${ }^{1}$ Fraunhofer Institute Material and Beam Technology IWS, Dresden, Germany \\ ines.daniliws.fraunhofer.de \\ ${ }^{2}$ Technical University Dresden, Germany
}

\begin{abstract}
Waste heat in the temperature range up to $100^{\circ} \mathrm{C}$ can be found in virtually all areas of industry and building and is often the hardest to recover costefficiently. It accounts for more than $50 \%$ of the total heat generated in industry [1].

Therefore, increasing interest is noticed in thermoelectric harvesters in the operation temperature range from room temperature up to $100^{\circ} \mathrm{C}$. The aim of this work is the development of materials and processes for the material and cost efficient scalable production of TEGs.

PEDOT:PSS is an interesting p-type conducting polymer suitable for printing of TEGs. A printed unileg generator, i.e. by using just a p-conductive material, was realised by dispenser printing of DMSO-doped PEDOT:PSS on polyimide. Contacts were made from silver. A TEG with 60 legs was characterized. The internal resistance is increasing linearly with the number of legs, confirming the good reproducibility of the printing process. By using a multilayer design the resistance can be decreased by more than $50 \%$. A temperature difference of 90 $\mathrm{K}$ results in a voltage of $37 \mathrm{mV}$ (at $20^{\circ} \mathrm{C}$ at the cold side).
\end{abstract}

\section{$1 \quad$ Introduction}

In developed countries, ca. $40 \%$ of the total fuel consumption is used for heating. Of this, about one-third is wasted due to insufficient transformation technologies into electricity. This wasted heat may be used by exploiting the Seebeck effect, enabling special materials to generate electrical energy if exposed to a temperature gradient. Thermoelectric generators (TEGs) built up from these materials are a durable way for energy supply without any moving components. Drawbacks of state-of-the-art materials are their low efficiency, limited availability or toxicity of the raw materials, and high costs.

Polymer materials in combination with printing techniques offer the possibility to manufacture flexible generators from non-toxic and easily available raw materials. The operation temperature of these materials ranges from room temperature up to 100 ${ }^{\circ} \mathrm{C}$; small temperature gradients of $1 \mathrm{~K}$ can be exploited.

adfa, p. 1, 2011.

(C) Springer-Verlag Berlin Heidelberg 2011 


\section{Material development}

PEDOT:PSS (poly(3,4-ethylenedioxythiophene) poly(styrenesulfonate) is an intrinsically p-type conductive polymer and possesses a high conductivity, good stability and flexibility. All printing experiments presented here are based on the printing paste SV3 (Heraeus) with a viscosity of 1 Pa.s (at a shear rate of $250 \mathrm{~s}-1$ ). The solids content is about $2.3 \mathrm{wt} \%$.

Due to the high viscosity of the PEDOT:PSS printing paste a dispenser printer (Asymtec) was used (figure 1).

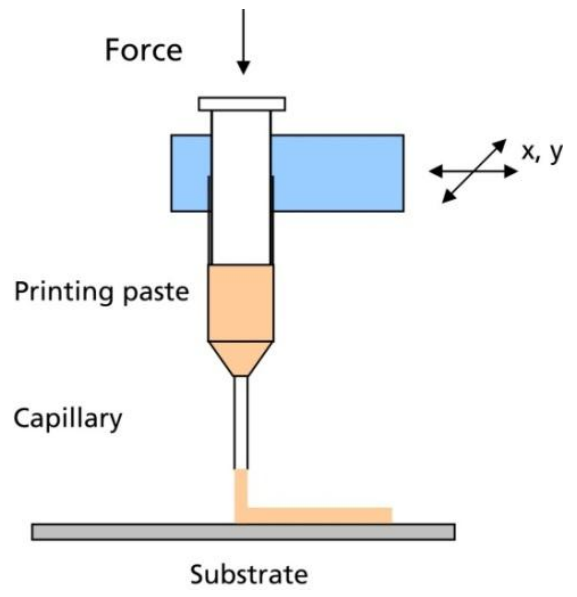

Fig. 1. Scheme of the dispenser printing process

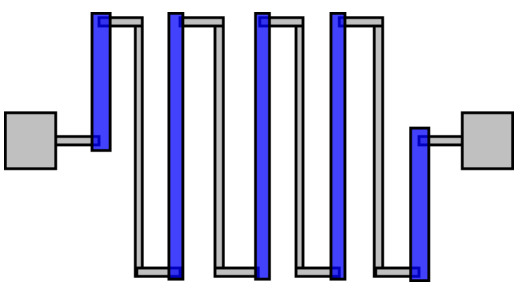

Fig. 2. Scheme of an unileg TEG - p-type conductive thermoelectric material (blue) and silver contacts (grey)

To increase the charge carrier concentration of the polymer, PEDOT:PSS was doped with dimethyl sulfoxide (DMSO). For characterisation of the thermoelectric properties the pastes were printed on glass substrates and dried at $50^{\circ} \mathrm{C}$ for 2 days. The resulting films with a thickness of $22 \mu \mathrm{m}$ were tested with a modified van der Pauw method [2]. The electrical conductivity increased from $8 \mathrm{~S} / \mathrm{cm}$ without DMSO addition to $84 \mathrm{~S} / \mathrm{cm}$ with $6 \mathrm{wt} \%$ DMSO. The Seebeck coefficient of about $15 \mu \mathrm{V} / \mathrm{K}$ was not influenced by DMSO doping. 


\section{$3 \quad$ Printing process and results}

Until now, no n-type conductive polymer for thermoelectric applications is commercially available. Therefore only p-type conductive PEDOT:PSS was used to print an unileg thermoelectric generator (figure 2).

Polyimide film was chosen as substrate due to its temperature stability up to 400 ${ }^{\circ} \mathrm{C}$ and its flexibility. In a first step, silver paste (Heraeus) was printed as contact material and sintered at $200^{\circ} \mathrm{C}$. PEDOT:PSS with $6 \mathrm{wt} \%$ DMSO serves as the active thermoelectric material. It was printed on top of the silver contact and dried at $60^{\circ} \mathrm{C}$ for $24 \mathrm{~h}$. A multilayer structure of the active material reduces the internal resistance by half, enabling a higher number of thermocouple legs with the same internal resistance.

Using a dispenser printing process enables a fast adaption of the unileg geometry; standard parameters are a length of $1 \mathrm{~mm}$ and a height of $10 \mathrm{~mm}$ for one unileg. A TEG with 60 unilegs corresponds to a total length of about $30 \mathrm{~cm}$ (figure 3).

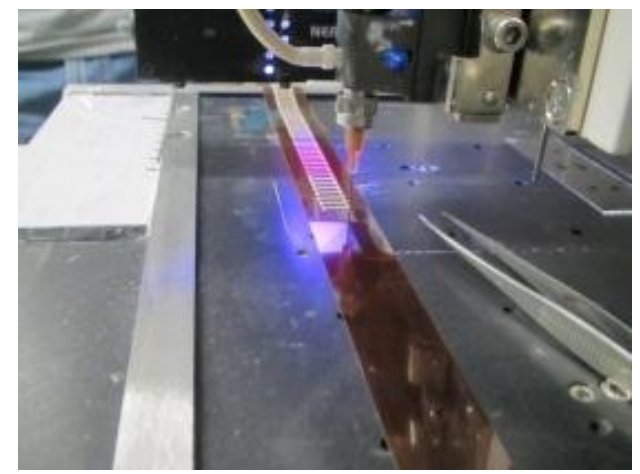

Fig. 3. Dispenser printing process of a TEG with 60 unilegs

For analyzing the thermoelectric performance of the device, the printed unileg film was wrapped around an adapter, made from two aluminum parts and a PEEK connector with low heat conductivity (figure 4).
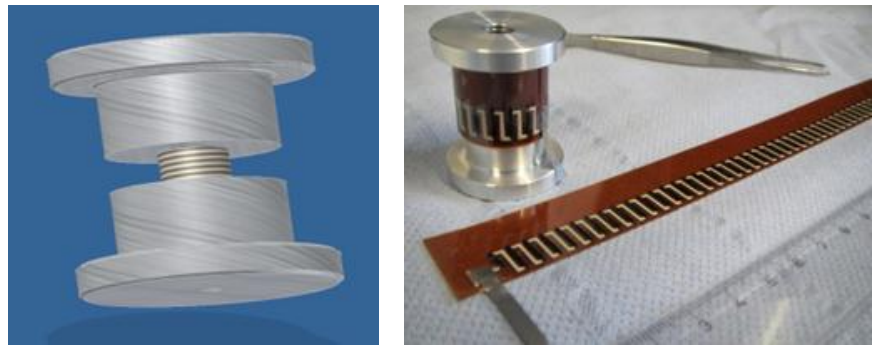

Fig. 4. CAD model of the adapter for winding-up the unileg film (left) and complete TEG with contacts (right) 
The cold side was held constant at $20^{\circ} \mathrm{C}$, the temperature of the hot side was stepwise increased. Increasing the temperature gradient leads to a linear increase in the measured voltage (figure 5). For a temperature difference of $90 \mathrm{~K}$ a resulting voltage of 37 $\mathrm{mV}$ was determined.

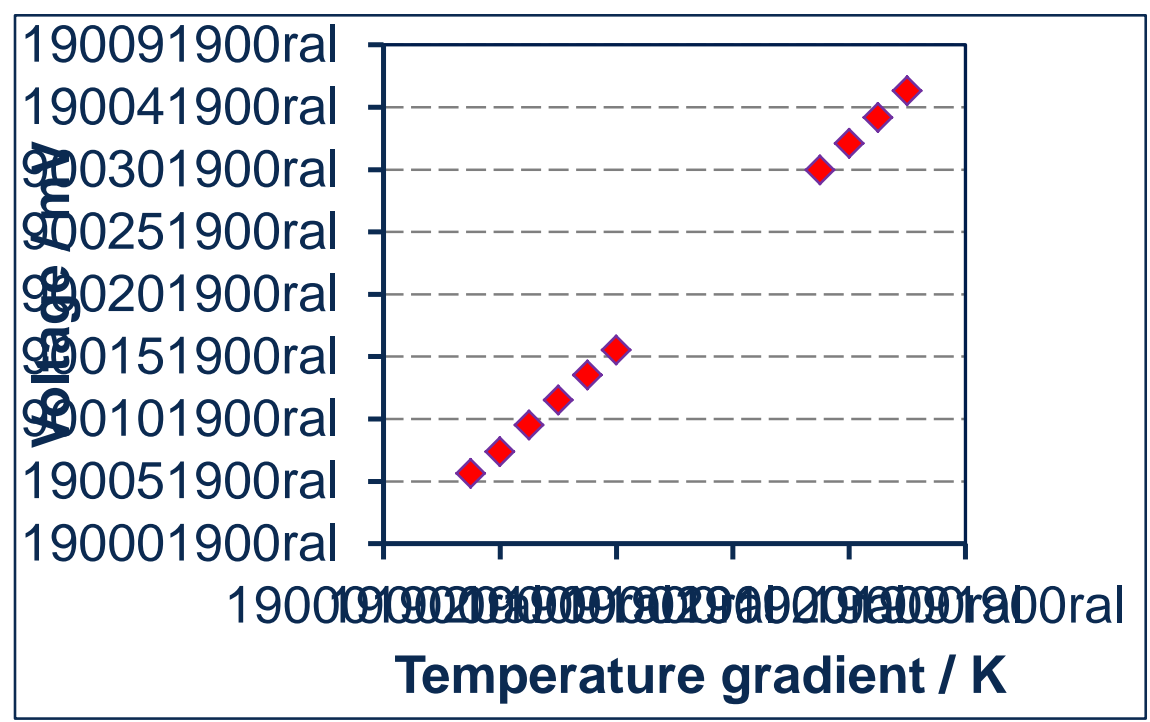

Fig. 5. Voltage in dependence of the temperature gradient in the TEG, cold side at $20^{\circ} \mathrm{C}$

\section{Summary}

A printed unileg generator consisting of only a p-conductive material was realised by dispenser printing of DMSO-doped PEDOT:PSS on polyimide to demonstrate the manufacturing of flexible thermoelectric generators from non-toxic and easily available raw materials. A TEG with 60 legs and silver contacts was characterized. By using a multilayer design the internal resistance was decreased by more than $50 \%$. With a cold side temperature of $20^{\circ} \mathrm{C}$ and a temperature difference of $90 \mathrm{~K}$ a voltage of 37 $\mathrm{mV}$ was generated.

\section{$5 \quad$ References}

1. S. Quoilon, Appl. Thermal Engineering, 31 (2011) 2885-2893

2. J. De Boor, V. Schmidt, Adv. Materials 22 (2010), 4303-4307 